\title{
Sclerosing Polycystic Adenosis of Salivary Gland: A Newly Described Tumor with Review of Literature
}

\author{
Shweta Rathi, Wasim Selimul Haque, Vinita Pant, Anita Borges \\ SRL Diagnostics, Centre of Excellence, Histopathology, Mahim, Mumbai, Maharashtra, India.
}

\section{Corresponding Author:}

Dr Shweta Rathi

Email: drshwetamantri12@gmail.com

This is an Open Access article distributed under the terms of the Creative Commons Attribution License (creativecommons.org/ licenses/by/3.0).

Received Accepted

Published

November 5, 2020

December 24, 2020

February 28, 2021

\begin{abstract}
Background: Sclerosing polycystic adenosis (SPA) is a rare, recently described, tumorous condition of the major or minor salivary glands that on gross and microscopic evaluation mimics an invasive carcinoma. The histology is quite similar to that seen in fibrocystic disease, sclerosing adenosis and adenosis tumors of the breast. It also resembles the more diffuse and bilaterally occurring polycystic dysgenetic disease (PDD) of the parotid gland. To the best of our knowledge, only 68 cases have been described so far. Case Report: Here we describe a case of SPA involving the parotid gland in a 58 year old male. At the first instance, the possibility of a low grade mucoepidermoid carcinoma was considered. However, histologically, it was a well circumscribed lobulated lesion composed of acini and ducts in a sclerotic stroma. Immuno-staining for SMA and p63 highlighted the myoepithelial cells around the ducts and acini which exhibited extensive metaplastic changes. Conclusion: Awareness of SPA is essential to avoid misdiagnosis with other common malignant salivary gland tumors and thus prevent its overtreatment.
\end{abstract}

Keywords: Diagnostic Errors, Hyperplasia, Mucoepidermoid Carcinoma, Parotid Gland, Salivary Gland Neoplasms.

\section{Introduction}

Sclerosing polycystic adenosis (SPA) is a rare, nodular, tumorous lesion that has a definite morphological similarity to fibrocystic changes, sclerosing adenosis and adenosis tumors of the breast. It also resembles the more diffuse and bilaterally occurring polycystic dysgenetic disease of the parotid gland. Smith et al. first reported SPA in 1996 [1]. To the best of our knowledge, 68 cases have been described so far [2]. Because of its rarity, SPA is a diagnostic challenge.

\section{Case Report}

A 58-year-old male presented with a swelling in his left cheek for one year. The pre-operative diagnostic work-up consisted of a magnetic resonance imaging (MRI) scan of the region, and fine needle aspiration cytology (FNAC). MRI reported it to be a well-defined, abnormal, single, solid, space occupying lesion in the superficial lobe of the left parotid gland, minimally bulging into the deep lobe. It measured approximately $3.6 \times 3.1 \times 2.9 \mathrm{~cm}$ in size. The FNAC was compatible with pleomorphic adenoma. A superficial parotidectomy along with dissection of levels I to III cervical lymph nodes was performed. Slides and blocks were received in consultation at SRL Diagnostics, Centre of Excellence (SRL, COE). Gross examination by the referring pathologist recorded that the lesion was well circumscribed and measured $3 \times 3 \times 2.5 \mathrm{~cm}$ with a firm, fibrous cut surface along with a peripheral rim of normal salivary gland tissue. Histologically, it was a circumscribed lobulated lesion [Fig.1] composed of acini and ducts with pseudo-infiltrative pattern in a sclerotic stroma [Fig.2]. Overall, the low power appearance of the lesion closely resembled that of fibrocystic disease 
of the breast because of the presence of many dilated ducts and adenosis coupled with dense fibrosis of the stroma.

The lobules showed adenosis, with closely packed duct lined variably by flattened or low cuboidal epithelium with dense eosinophilic or clear cytoplasm together with mucous cells and vacuolated cells. Many dilated ducts, some of which were filled with luminal secretions were noted. Occasional clear cells are also present in the lining. The PAS stain highlighted luminal secretions and the absence of mucin in the clear cells [Fig.3]. Focally, acinic cell hyperplasia and cribriform intraductal proliferation were noted. Areas of infarction and squamous metaplasia were seen. The stroma showed a moderate perilobular chronic inflammatory infiltrate. The adjacent parotid gland was histologically unremarkable. Immunostaining for alpha smooth muscle actin (SMA), and p63 highlighted the myoepithelial cells around every duct and acinus [Fig.4].

\section{Discussion}

Sclerosing polycystic adenosis (SPA) is a rare condition primarily of the major salivary glands. The distribution of involvement is parotid gland (71\%), submandibular gland $(8 \%)$, minor salivary glands in the oral cavity (13\%) and nasal mucosa $(3.2 \%)$. A case of SPA affecting the lacrimal gland has also been reported [3]. SPA shows no gender predilection and occurs over a wide age spectrum of 7-84 years [2]. The size of SPA lesions ranges from $3 \mathrm{~mm}$ (incidental SPA) to $6 \mathrm{~cm} \mathrm{[2].}$

The etiology of SPA is still speculative. Skálová et al. demonstrated a clonal biology of SPA and concluded that SPA is a neoplasm and not just a reactive process based on a $30 \%$ recurrence rate with hyperplastic, atypical and dysplastic microscopic features [5]. Swelam et al. reported three cases of SPA which were associated with the Epstein Barr virus [6]. SPA is not included as a tumor in the current WHO classification but was

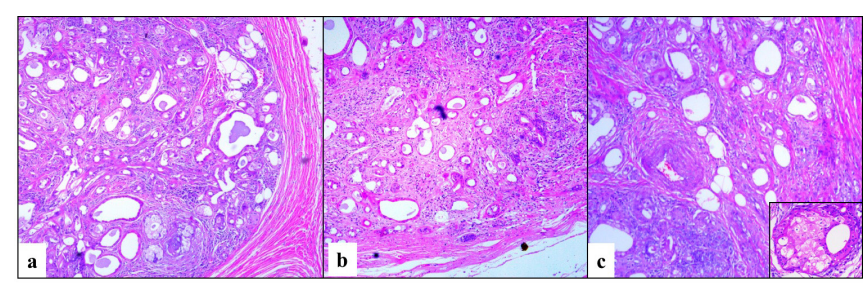

Fig.1: A well circumscribed and lobulated lesion with adenosis, cystic change - overall low power appearance mimicking that of fibrocystic disease of breast. Intraluminal secretions are also present in some of the ducts; $H$ and $E 20 \times$ (Inset-Apocrine appearance).

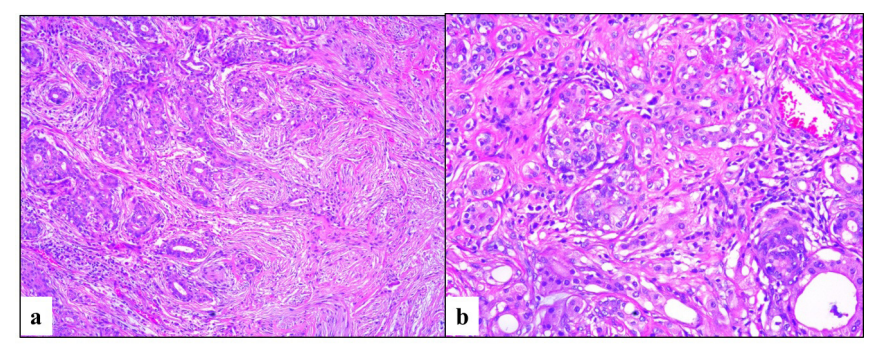

Fig.2: Pseudo-infiltrative pattern within the lobule ( $H$ and $E$ $10 \times$ and $40 \times)$.

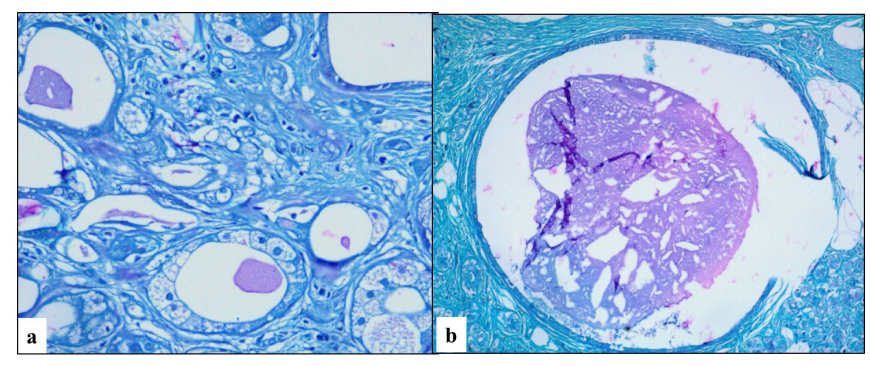

Fig.3: PAS stain highlights the absence of mucin within the vacuolated cells lining cystic spaces.

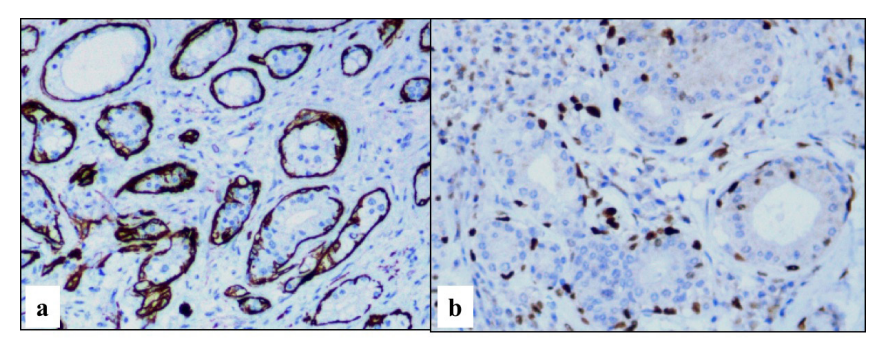

Fig.4: Intact SMA and p63 expressing myoepithelial layer around the tubules; p63 does not mark the lining epithelial cells ruling out mucoepidermoid carcinoma (immunohistochemical $400 \times$ ). 
included in the chapter on Tumor-like Conditions of the AFIP Series 4 in Atlas of Tumors of the Salivary Glands [7].

The largest series to date was published by Gnepp et al. which comprised 16 new cases and a review of 31 previously reported cases [4]. It has a favorable outcome with no deaths reported to date. Recurrences have been reported varying from 19 to $30 \%$ [2,5]. The proposed mechanism for the recurrences is either incomplete surgical resection and/or multifocal disease. However, additional investigations and longer follow-up periods are necessary to further clarify the nature of the lesion. The differential diagnostic considerations for SPA have been discussed and commented on by previous authors which include acinic cell carcinoma (ACC), sclerotic sialadenitis, polycystic dysgenetic disease (PDD), and a low-grade mucoepidermoid carcinoma $[1,4,8,9]$. In SPA, lobularity is typically maintained as observed in similar lesions in the breast viz. fibrocystic breast disease, sclerosing adenosis and adenosis tumors. The acinic cell and ductal proliferation of SPA may suggest the possibility of an acinic cell carcinoma, especially in cases with prominent atypical ductal proliferation in the regions of adenosis. However, in SPA the lobular architecture is typically maintained along with the myoepithelial cell layer and there is a lack of the destructive growth pattern of a carcinoma. Sclerosing sialadenitis has prominent fibrosis with chronic inflammation; however, unlike SPA, the fibrosis does not form nodules, the parenchyma is atrophic without ductal or acinar hyperplasia and the cystic changes are usually minimal.

PDD has a lattice-like network of cysts with inspissated intra-cystic secretions replacing normal parenchyma with only small intervening clusters of residual acini. However, fibrosis is not prominent and ductal or acinar proliferation is absent. Moreover, PDD is a very rare condition of developmental origin commonly affecting the both parotid glands although unilateral involvement of the parotid is also on record $[10,11]$. In the present case there is a rich ductal proliferation along with an intraductal cribriform proliferation in addition to the presence of cystically dilated ducts, histologic features more in favor of SPA than PDD. The striking similarity to PDD suggests that SPA may represent a localized form of this dysgenetic condition that persists into adulthood and undergoes additional proliferative and even neoplastic change.

Low-grade mucoepidermoid carcinoma (MCA) was a close differential diagnosis with an overall appearance of the lesion being pseudoinfiltrative reflected by ducts of various sizes and shapes, some dilated and some irregularly contoured, in a fibrous stroma. The presence of inspissated secretions in the ductal lumen and clear cells lining a few of the ducts was also reminiscent of MCA. However, intra-cytoplasmic mucin could not be demonstrated in the tumor. The presence of foci of infarction was also ominous looking. But the overall low power view resembling that of fibrocystic disease of the breast, with retained lobular appearance gave us the first indication that we were not dealing with an infiltrative carcinoma. Furthermore, histological features like nuclear atypia, mitotic activity, and perineural invasion were absent. Features like dense sclerosis, cystic changes and adenosis, which are consistently seen in SPA were present. Subsequent immunohistochemical evaluation highlights intact myoepithelial layer by $\mathrm{p} 63$ and SMA. MCA representing a tumor of the excretory ducts does not exhibit a consistent abluminal myoepithelial lining.

Superficial parotidectomy is recommended for initial lesions and total parotidectomy for recurrent ones [12]. Matsumoto et al. analyzed surgery-dependent recurrence rates and found them to be $44.4 \%, 7.1 \%$, and $10 \%$ for enucleation/ excision, superficial parotidectomy and total parotidectomy, respectively [13]. They suggest that parotidectomy can yield a lower recurrence rate as compared to enucleation/excision. 


\section{Conclusion}

We have described a case of SPA which was initially mistaken for a MCA and reviewed the literature on this benign lesion, its differential diagnosis, behavior and speculations as to its nature. Its morphologic mimicry to invasive carcinoma coupled with its rarity may lure an unwary histopathologist to overcall the case as a carcinoma. The lobular nature of the lesion on low power is an important clue to the histologic diagnosis as it is with many benign lesions of the breast. We speculate that given its resemblance to PDD, SPA may represent a persistent, localized form of salivary gland dysgenesis, which in adulthood may acquire proliferative and possibly neoplastic features. At one year of follow up, the patient is asymptomatic, with a favorable clinical outcome and no evidence of recurrence.

Contributors: SR: Manuscript editing, and histopathology; WSH: manuscript editing, references and histopathology; VP, AB: critical inputs into the manuscript and histopathology. SR will act as a study guarantor. All authors approved the final version of this manuscript and are responsible for all aspects of the study.

Funding: None; Competing interests: None stated.

\section{References}

1. Smith BC, Ellis GL, Slater LJ, Foss RD. Sclerosing polycystic adenosis of major salivary glands. Am J Surg Pathol. 1996;20:161-170.

2. Appasani S, Ramadas P, Gangadharan J, Mathews A. Sclerosing polycystic adenosis: A case report with review of the literature. Indian Journal of Pathology and Microbiology. 2019;62:136-138.

3. Pfeiffer ML, Yin VT, Bell D, Mancini R, Esmaeli B. Sclerosing polycystic adenosis of the lacrimal gland. Ophthalmology. 2013;120:873-873.e1.
4. Gnepp DR, Wang LJ, Brandwein-Gensler M, Slootweg P, Gill M, Hille J. Sclerosing polycystic adenosis of the Salivary Gland. Am J Surg Pathol. 2006;30:154-164.

5. Skálová A, Gnepp DR, Simpson RHW, Lewis JE, Janssen D, Radek Sima R, et al. Clonal nature of sclerosing polycystic adenosis of salivary glands demonstrated by using the polymorphism of the human androgen receptor (HUMARA) locus as a marker. Am J Surg Pathol. 2006;30:939-944.

6. Swelam WM. The pathogenic role of Epstein-Barr virus (EBV) in sclerosing polycystic adenosis. Pathol Res Pract. 2010;206:565-571.

7. AntonescuWMC, Scheithauer BW, Woodruff JM. Tumors of the Peripheral Nervous System: AFIP Atlas of Tumor Pathology, Series 4 ARP Press, Silver Spring, MD, 2013.

8. Skálová A, Michal M, Simpson RHW, Stárek I, Prádná J, Pfaltz M. Sclerosing polycystic adenosis of parotid gland with dysplasia and ductal carcinoma in situ. Report of three cases with immunohistochemical and ultrastructural examination. Virchows Archiv. 2002;440:29-35.

9. Gnepp DR. Sclerosing polycystic adenosis of the salivary gland: A lesion that may be associated with dysplasia and carcinoma in situ. Advances in Anatomic Pathology. 2003;10:218-222.

10. Kumar KRA, Mahadesh J, Setty S. Dysgenetic polycystic disease of the parotid gland: Report of a case and review of the literature. J Oral Maxillofac Pathol. 2013;17:248252.

11. Barnes L. Surgical Pathology of the Head and Neck, Third Edition. CRC Press, 2008.

12. Mackle T, Mulligan AM, Dervan PA, Mulligan AM, Dervan PA, O'Dwyer T. Sclerosing polycystic sialadenopathy- a rare cause of recurrent tumor of the parotid gland.Arch Otolaryngol Head Neck Surg. 2004; 130:357-360.

13. Matsumoto NM, Umezawa H, Ohashi R, Peng WX, Zenya Naito, Ogawaet R. Surgical treatment of rare sclerosing polycystic adenosis of the deep parotid gland. PlastReconstr Surg Glob Open. 2016;4(3):e645. 